\title{
Does Hyponatremia have a Value in the Diagnosis of Extrapulmonary Tuberculosis in HIV-1 Infected Patients in Cambodia?
}

\author{
Lut Lynen*,1, Sok Phan ${ }^{2}$, Suos Prem Prey ${ }^{3}$, Thai Sopheak ${ }^{2}$, Joseph Harwell ${ }^{4}$, Marleen Boelaert ${ }^{1}$, \\ Olivier Koole ${ }^{1}$, Robert Colebunders ${ }^{1,5}$ \\ ${ }^{1}$ Institute of Tropical Medicine, Antwerp, Belgium \\ ${ }^{2}$ Sihanouk Hospital Center of HOPE, Phnom Penh, Cambodia \\ ${ }^{3}$ NCHADS, National Center for HIV/AIDS Dermatology and STDs \\ ${ }^{4}$ The Warren Alpert Medical School at Brown University, division of infectious diseases, departments of internal medi- \\ cine and pediatrics, Brown University, Providence, Rhodes Island, USA
}

${ }^{5}$ University of Antwerp, Antwerp, Belgium

\begin{abstract}
Hyponatremia is a frequent finding in hospitalised HIV-patients. The diagnosis of extra-pulmonary tuberculosis (EPTB) is difficult in resource-limited settings, causing delays in treatment. In this cross-sectional study based on hospital chart review in a charity hospital in Cambodia we found that hyponatremia (serum sodium $<134 \mathrm{meq} / \mathrm{L}$ ) was significantly associated with a diagnosis of EPTB in HIV-positive adults. Our findings suggest that hyponatremia in patients with advanced stage of HIV disease should trigger a diagnostic work-up for EPTB.
\end{abstract}

\section{INTRODUCTION}

Worldwide more than 40 million people are HIVinfected, and over half of them are co-infected with tuberculosis (TB). Diagnostic facilities are often limited in resourceconstrained settings causing delays in diagnosis of smear negative pulmonary TB (PTB) and extra-pulmonary TB (EPTB). This leads to avoidable deaths. Therefore the "Stop TB Strategy" has issued new guidelines for the timely diagnosis and treatment of smear-negative and extra-pulmonary TB [1].

The World Health Organisation (WHO) has classified Cambodia as one of the 22 high burden countries with TB in the world. Annual TB incidence rate of all forms is estimated at 508/100,000 inhabitants [2]. In 2002, at the time of this study, the HIV prevalence in Cambodia was the highest in Asia, with $2.6 \%$ in the age group 15-49 year-old, in the general population [3]. In 2005 the HIV seroprevalence rate among TB patients was about $10 \%$ [4]. A retrospective chart review of 299 HIV positive outpatients and inpatients in the Sihanouk Hospital Center of HOPE (SHCH) in Phnom Penh (March 1999-June 2000) showed that 49\% of the patients had TB, the majority (62\%) being EPTB [5]. Hyponatremia is a frequent finding in hospitalized patients with HIV infection. A study from the US showed that hyponatremia $(<135$ $\mathrm{mmol} / \mathrm{l}$ ) was found in $60 \%$ of AIDS patients with a diagnosis of generalized TB, but half of these cases of disseminated TB were only diagnosed post-mortem [6].

Hyponatremia is associated with opportunistic infections and with increased mortality [7]. This seems particularly true for patients with HIV-infection and disseminated TB [8] and has been associated with a syndrome of inappropriate antidiuretic hormone (SIADH). We studied the value of hypona-

\footnotetext{
*Address correspondence to this author at the Institute of Tropical Medicine, Antwerp, Belgium; E-mail: LLynen@itg.be
}

tremia as a marker for EPTB diagnosis among hospitalized patients with HIV-infection at the SHCH.

\section{METHOD}

Study setting: The $\mathrm{SHCH}$ is a charity hospital for adults in Phnom Penh, Cambodia, which opened in November 1996. The hospital provides free and standard medical care to the poor and needy. Due to the limited number of hospital beds (only 23 beds), most patients receive ambulatory care. Approximately 5,000 patients are routinely seen at the hospital each month and most of these patients are self-referred to the hospital. HIV ambulatory care was established at the hospital in mid-1998. The clinic provided basic HIV testing and counselling, clinical diagnosis and treatment of opportunistic infections. CD4 count and antiretroviral therapy were not yet available. Between 1999 and 2001, an estimated 250 HIV-infected patients were seen each month at the outpatient clinic. Of these, 30-40 were newly detected HIV positive patients.

Study design: a cross-sectional study based on chart review of all HIV patients who had been hospitalised between January 1998 and December 2001. Only first time admissions were considered.

EPTB patients were defined as patients with at least one of the following: scrofula, abdominal lymphadenitis diagnosed with abdominal ultrasound, abnormal spine X-ray (Pott's disease), abnormal chest X-ray showing a pleural or pericardial effusion or miliary $\mathrm{TB}$, lymphocytic meningitis with slow onset, and a response to anti-tuberculous treatment (ATT). They were compared with HIV-positive patients with a first-time admission who had a different diagnosis than the ones mentioned above.

The following data were recorded on a standard case record form: sex, age, serum sodium and potassium (AVL 9130, AVL, Roswell, USA), creatinine and glycemia (Clin- 
line 150, BioMérieux SA, France) and blood pressure on admission, dyspnoea and hypoxia on admission, abdominal pain on admission, peripheral lymph nodes on physical exam, discharge diagnosis and treatment outcome. The Student's t-test was used to compare means of continuous variables such as age, serum sodium, creatinine, potassium in cases and controls. The optimal cut-off for hyponatremia to predict EPTB was determined by constructing a ROC curve and identifying the best cut-off as the value where the sum of the sensitivity and specificity was greatest.

The association between categorical variables and EPTB was examined using the Pearson Chi-square test without continuity correction, ROC-curves were constructed and area under the curve (AUC) was calculated. Variables with a good discriminative power (AUC >0.6) were retained.

Clinical relevant variables that were significant at an $\alpha$ $<0.10$ in the univariate analysis were included in a multivariate logistic regression analysis. The goodness of fit of the final model was checked with the Hosmer \& Lemeshow Test [9]. Interaction between covariates was examined by running an automatic forward stepwise logistic regression including interaction terms. Sensitivity and specificity were calculated and the Wilson score method without continuity correction was used to calculate $95 \%$ confidence intervals for sensitivity, specificity [10]. Diagnostic accuracy of the markers was further assessed by examining likelihood ratios and diagnostic odds [11].

To calculate the post-test odds we apply the Bayes theorem: post-test odds $=$ pre-test odds $\mathrm{x}$ LHR [11]. From the post-test odds the probability of a diagnosis of EPTB can be estimated in a patient with hyponatremia by applying the formula: odds $=\mathrm{P} / 1-\mathrm{P}$, where $\mathrm{P}$ is the probability of disease, given a certain test result [11].

Data were analyzed with SPSS 15.0 for Windows, SPPS Inc., Chicago, Illinois.

The research protocol was approved by the institutional review board of the SHCH.

\section{RESULTS}

123 patients fulfilled the eligibility criteria. Among them, 42 patients (34\%) had EPTB as a discharge diagnosis (see Table 1). Of these, 12 patients $(28.6 \%)$ had EPTB + PTB combined. Among the 81 controls, 29 patients $(35.8 \%)$ had PTB.

Table 1. Discharge Diagnosis in 123 HIV Patients Included in the Study

\begin{tabular}{|c|c|c|}
\hline Diagnosis & $\mathbf{n}^{*}$ & $\mathbf{\%}$ \\
\hline \hline EPTB & 42 & 34.1 \\
\hline PTB** $^{* *}$ & 41 & 33.3 \\
\hline PCP $^{\ddagger}$ & 58 & 47.2 \\
\hline Cryptococcal $^{\dagger}$ meningitis & 16 & 13 \\
\hline Other & 17 & 13.8 \\
\hline
\end{tabular}

*Patients can have more than 1 diagnosis, **12 patients have EPTB + PTB combined, ${ }^{\ddagger}$ Pneumocystis Jiroveci pneumonia, diagnosed clinically based on hypoxia and bilateral interstitial infiltrates, with response to high dose cotrimoxazole, "Diagnosed by the presence of encapsulated yeasts on Indian ink stain of CSF.
The different syndromes associated with the diagnosis of EPTB are shown in Table 2.

Table 2. Types of EPTB $(n=42) *$

\begin{tabular}{|c|c|c|}
\hline Syndromes & $\mathbf{n}$ & $\boldsymbol{\%}$ \\
\hline \hline Abdominal lymph nodes/ ascites/peritonitis & 23 & 54.8 \\
\hline Pleural/pericardial effusion & 13 & 30.9 \\
\hline Pott's disease & 12 & 28.6 \\
\hline TB meningitis & 11 & 26.2 \\
\hline Disseminated TB & 10 & 23.8 \\
\hline TB lympadenitis (scrofula) & 8 & 19.0 \\
\hline Miliary TB & 3 & 7.1 \\
\hline
\end{tabular}

*More than 1 diagnosis is possible.

Mean serum sodium was significantly lower in EPTB patients compared to others $(133 \mathrm{meq} / \mathrm{L}$ versus $139 \mathrm{meq} / \mathrm{L}$, ttest, $\mathrm{p}<0.001)$. Mean age, serum creatinine, serum potassium, blood pressure were not associated with a diagnosis of EPTB. The ROC curve resulted in an optimal cut-off for the diagnosis of EPTB at a sodium level $<134 \mathrm{Meq} / \mathrm{L}$ (AUC $0.71)$. At this cut-off, 39 (32\%) patients in our study showed hyponatremia. Hyponatremia was significantly more frequent in patients with EPTB than in patients with other discharge diagnoses ( $57 \%$ versus $18.5 \%$, Chi-square, $\mathrm{p}<0.001$ ). In the final logistic regression model hyponatremia, hypoxia on admission and male sex were significantly associated with EPTB (see Table 3). The Hosmer-Lemeshow Goodness of Fit test supports this model (chi-square=3.167; $\mathrm{p}=0.674$ ). There was no interaction between the covariates.

Hyponatremia defined as sodium $<134 \mathrm{meq} / \mathrm{L}$ had a sensitivity of $57 \%$ (95\%CI: $42-71 \%$ ) and a specificity of $81.5 \%$ (95\%CI: $72-88 \%$ ) to detect EPTB. The positive likelihood ratio (LHR+) was 3.09 (95\%CI: 1.8-5.2). A person with EPTB was 3 times more likely to have hyponatremia than a patient with no EPTB. The negative LHR (LHR-) was 0.5 (95\%CI: 0.4-0.7), giving a diagnostic odds (LHR+/LHR-) of 5.89 (95\%CI: 2.56-13.45). Applying the Bayes theorem the post-test odds for EPTB in case of hyponatremia can be calculated. Post-test odds $=$ pre-test odds $(42 / 81=0.52) \times$ LHR $(3.09)=1.6$. This represents a post-test probability of 0.62 .

\section{DISCUSSION}

$32 \%$ of our hospitalized patients with HIV infection presented with hyponatremia (sodium $<134 \mathrm{meq} / \mathrm{L}$ ), which is consistent with other reports who use $135 \mathrm{meq} / \mathrm{l}$ as the cut off $[12,13]$. In our hospital HIV population the pre-test probability of EPTB was 0.34 (42/123). Our results show that the probability of EPTB increases to 0.62 if a patient has hyponatremia.

However, causes of hyponatremia in patients with AIDS are multiple, volume depletion secondary to diarrhoea and syndrome of inappropriate antidiuretic hormone (SIADH) being the most important. In patients with SIADH Pneumocystis jiroveci pneumonia (PCP), bacterial pneumonia and central nervous system infections are described as causes [13]. Therefore, hyponatremia can be at best considered as a 
Table 3. Characteristics of EPTB Cases and Controls (n=123)

\begin{tabular}{|c|c|c|c|c|c|c|}
\hline & $\begin{array}{c}\text { EPTB cases } \\
\mathrm{n}=42(\%)\end{array}$ & $\begin{array}{c}\text { Controls } \\
\mathbf{n}=\mathbf{8 1}(\%)\end{array}$ & $\begin{array}{c}\text { Crude Odds ratio } \\
\qquad(95 \% \mathrm{CI})\end{array}$ & P-value * & $\begin{array}{l}\text { Adjusted Odds ratio } \\
(95 \% \text { CI })\end{array}$ & P-value ** \\
\hline Female sex & $30(71.4)$ & $45(55.6)$ & $0.5(0.21-1.19)$ & 0.087 & $0.36(0.13-0.95)$ & 0.039 \\
\hline Sodium $<134 \mathrm{meq} / 1$ & $24(57.1)$ & $15(18.5)$ & $5.87(2.37-14.71)$ & $<0.001$ & $6.39(2.38-17.18)$ & $<0.001$ \\
\hline Abdominal pain & $23(54.8)$ & $30(37.0)$ & $2.0(0.90-4.71)$ & 0.060 & & \\
\hline Hypoxia on admission & $2(4.9)$ & $39(31.7)$ & $0.05(0.01-0.25)$ & $<0.001$ & $0.039(0.008-0.194)$ & $<0.001$ \\
\hline Diarrhoeal disease & $12(28.6)$ & $25(30.9)$ & $0.90(0.36-2.19)$ & $>0.2$ & & \\
\hline
\end{tabular}

*Pearson Chi-square, **Wald-test in final logistic regression model.

non-specific marker of the EPTB syndrome that identifies the patients with very advanced disease. Nonetheless, it can be useful in clinical practice in resource constrained settings as a reminder to clinicians to consider the possibility of EPTB if hyponatremia is present. In our study setting in Cambodia it was significantly more present in EPTB than in other opportunistic infections. In particular PCP was not associated with hyponatremia, and this was also found in another study in Spain [8].

There are several limitations to our study. First the limited diagnostic capacity of the SHCH hospital did not allow for a microbiological confirmation of the diagnosis of EPTB and several other conditions. Secondly, the determination of possible causes of hyponatremia by performing cortisol or ACTH levels or urine chemistry was also not possible. Thirdly, our study population was small with many patients with concomitant infections. Fourthly, this is a crosssectional study based on a retrospective review of hospital chart data collected during routine clinical care. Investigations were done according the clinical presentation and were not a systematic set as one would perform in a prospective study. Therefore, it is possible that some diagnosis were overlooked. Finally, CD4 count results were not available. Therefore we cannot exclude that the correlation we observed between hyponatremia and EPTB was in fact explained (confounded) by the association with more advanced disease stage and not necessarily with EPTB itself.

Certainly, to confirm that ETPB is associated with hyponatriemia, additional studies, in other larger patient populations will be needed. Such studies should include more indepth investigations to determine the cause of hyponatremia in hospitalized patients with HIV infection in countries with limited resources. Nevertheless we suggest that in patients with WHO stage 3 or 4 , who are eligible for highly active antiretroviral treatment (HAART), and who present with atypical symptoms, serum sodium should be determined before the start of HAART. The detection of hyponatremia should trigger a diagnostic work-up for EPTB.

\section{CONCLUSION}

In adult hospitalized HIV-patients in Cambodia, hyponatremia (serum sodium < $134 \mathrm{Meq} / \mathrm{l}$ ) is associated with a diagnosis of extra-pulmonary TB. The presence of hyponatremia in patients with advanced stage of HIV disease should trigger a diagnostic work-up for EPTB.

\section{REFERENCES}

[1] STOP TB Department, Department of HIV/AIDS. Improving the diagnosis and treatment of smear-negative pulmonary tuberculosis among adults and adolescents. Recommendations for HIVprevalent and resource-constrained settings. WHO/HTM/TB/2007. 379/. 2007. WHO.

[2] Ministry of Health Cambodia. National Health Policies and Strategies for Tuberculosis Control (2006-2010). 2006.

[3] NCHADS. HIV Sentinel Surveillance. 2002. Cambodia, Ministry of Health.

[4] Ministry of Health Cambodia. Tuberculosis Report 2005. 2005.

[5] Sok, P.; Harwell, J.I. Clinical characteristics and outcomes of 299 HIV-infected patients at Sihanouk Hospital Center of HOPE (SHCH), Phnom Penh, Cambodia. XIVth International AIDS conference, Barcelona 7-12 July 2002.

[6] Smith, M.B.; Boyars, M.C.; Veasey, S.; Woods, G.L. Arch. Pathol. Lab. Med., 2000, 124, 1267-74.

[7] Cusano, A.J.; Thies, H.L.; Siegal, F.P.; Dreisbach, A.W.; Maesaka, J.K. J. Acquir. Immune. Defic. Syndr., 1990, 3, 949-53.

[8] Salqado, R.; Moya, M.S.; Barbadillo, R.; Calabrese, S.K.; Marchena, M.J. Hyponatremia and disseminated tuberculosis in AIDS. International Conference on AIDS, Jun 6-11, abstract no. PO-B07-1151.

[9] Field, A. In Discovering Statistics Using SPSS. $2^{\text {nd }}$ Edition. Chapter 6: Logistic Regression. Sage Publications Ltd, 2005, pp. 218-268.

[10] Newcombe, R.G. Two-sided confidence intervals for the single proportion: comparison of seven methods. Stat. Med., 1998, 17, 857-72.

[11] Hunink, M.; Glasziou, P.; Siegel, J.; Weeks, J.; Pliskin, J.; Elstein, A.; Weinstein, M. In Decision making in health and medicine. Chapter 5: Interpreting diagnostic information. Cambridge University Press, 2001, pp .128-156.

[12] Agarwal, A.; Soni, A.; Ciechanowsky, M.; Chander, P.; Treser, G. Nephron, 1989, 53, 317-21.

[13] Tang, W.W.; Kaptein, E.M.; Feinstein, E.I.; Massry, S.G. Am. J. Med., 1993, 94, 169-74. 